\title{
Comparison of Headspace Techniques for Sampling Volatile Natural Products in a Dynamic System
}

Jenny Fäldt ${ }^{\mathrm{a}, *}$, Mikael Eriksson ${ }^{\mathrm{b}}$, Irena Valterovác ${ }^{\text {and Anna-Karin Borg-Karlson }}{ }^{\mathrm{a}}$

a Department of Chemistry, Organic Chemistry, Ecological Chemistry, KTH,

SE-10044 Stockholm, Sweden. Fax: 4687912333. E-mail: akbk@kth.se

b Department of Biotechnology, Group Microbiology, KTH, SE-10044 Stockholm, Sweden

c Institute of Organic Chemistry and Biochemistry, Academy of Sciences of the

Czech Republic, Flemingovo nám. 2, 16610 Prague 6, Czech Republic

* Author for correspondence and reprint requests

Z. Naturforsch. 55c, 180-188 (2000); received November 25, 1999/February 10, 2000

Volatile Compounds, Dynamic Headspace, Sampling Technique

Commonly used dynamic sorption techniques for collecting biologically active volatile compounds have been compared. Solid phase microextraction (SPME) using two types of fibers (polydimethylsiloxane, PDMS, $100 \mu \mathrm{m}$, and carbowax/divinylbenzene, CW/DVB, 65 $\mu \mathrm{m})$ was compared to purge and trap methods (Porapak Q, Tenax TA and charcoal) and a technique based on absorption in methanol in a cooling bath. Sampling was done in a stream of purified air $(20 \mathrm{ml} / \mathrm{min})$ in a closed and temperature-regulated $\left(27^{\circ} \mathrm{C}\right)$ glass tube, passing over a capillary tube containing a hexane solution of tridecane, heptadecane, 1-octen-3-ol, 1hexadecanol, ethyl tetradecanoate, $\alpha$-pinene, linalool, terpinen-4-ol, cis-verbenol, verbenone, $\beta$-caryophyllene, $E, E$-farnesol, and geranylgeraniol. With all of the methods, the sampling was performed for a period of $30 \mathrm{~min}$ before extraction and analysis was done on a GC-FID system. In general, SPME gave a higher response for all compounds except for $\alpha$-pinene, which was only extracted by the CW/DVB fiber. Purge and trap methods and methanol absorption gave the same response for all substances extracted. None of the methods extracted hexadecanol and geranylgeraniol under the conditions used. However, the SPME equipped with the PDMS coating extracted heptadecane, $E, E$-farnesol and ethyl tetradecanoate. Our results show that SPME, when selecting the fibers to fit the polarity and volatility of the compounds, is an outstanding extraction method compared to purge and trap and methanol absorption, especially for a qualitative analysis. The best conditions for storing fibers exposed to compounds of high volatility were at low temperatures $\left(6^{\circ} \mathrm{C}\right)$ in sealed vials, while the worst way was to leave the exposed fiber unprotected at room temperature $\left(22^{\circ} \mathrm{C}\right)$. The dynamic sampling system was effectively tested on a fruiting body of a polypore fungus (Ganoderma applanatum) emitting 1-octen-3-ol, and again SPME showed to be the most sensitive technique.

\section{Introduction}

In ecological chemistry, e.g. in pollination chemistry, insect-plant relations, pheromone chemistry and in chemotaxonomy (Borg-Karlson, 1990; Knudsen, et al., 1993; Borg-Karlson and Mozuraitis, 1996) efficient tools for sampling volatile compounds are needed. To understand the communication systems among organisms it is necessary to collect volatile semiochemicals in sufficient amounts without disturbing, and to determine both structures and quantities of the behaviorally active compounds (Malosse et al., 1995). A careful selection of extraction techniques is important for minimizing the loss of compounds during the collection. Behaviorally active compounds (BAC) are often produced in mixtures at low concentrations (ng levels) and it is important to handle the samples with care to avoid contamination. Large numbers of samples are often needed for statistical calculations, to serve as bases for general conclusions. A common extraction technique for sampling of BAC, is headspace sampling in air streams using purge and trap with different adsorption materials (Porapak, Tenax or charcoal) (Agelopoulos and Pickett, 1998; Cross et al., 1976). Other ways of extracting volatile compounds from a gas phase is to use a liquid sorbent trap, e.g. methanol, cooled with $\mathrm{CO}_{2} /$ ethanol $\left(-78^{\circ} \mathrm{C}\right)$. These techniques are well established and widely used. However, techniques are constantly being modified and a relatively new one, which has been accepted more and more during the last years, is the solid phase microextraction (SPME). This is a solvent-free ex- 
traction method (Pawliszyn, 1997), based on a fused silica fiber coated with polymers, such as polydimethylsiloxane or Carbowax/divinylbenzene, which are attached to a GC-syringe. SPME has been used with success for the extraction in air streams (Bartelt and Zilkowski, 1999; Eisert et al., 1998), and by static samplings (Matich, et al., 1996; Miller and Stuart 1999; Eriksson et al., 1998), and analysis of numerous organic compounds from various environments.

In this study, headspace SPME using two types of fibers was compared with conventional purge and trap methods, using three adsorbents (Porapak Q, Tenax TA and charcoal), and with adsorption in methanol in a cold trap. A standard mixture of compounds, commonly occurring in insect communication, was extracted in a dynamic system (Jursik et al., 1991) using purified air. The dynamic sampling system was also tested on a natural sample, the polypore fungus Ganoderma applanatum, emitting 1-octen-3-ol. This was done for the purpose of comparing the sorption obtained by these methods, their ease of use and their effectiveness. In addition, possibilities of storing fibers at different temperatures were investigated, giving useful information for sampling in situ.

\section{Methods and Materials}

\section{Chemicals}

The compounds used were chosen to represent a broad range in vapour pressure and to represent commonly occurring semiochemicals (Table I); tridecane, heptadecane, 1-octen-3-ol, 1-hexadecanol, ethyl tetradecanoate, $\alpha$-pinene, linalool, terpinen4-ol, cis-verbenol, verbenone, E,E-farnesol, and geranylgeraniol from Sigma-Aldrich (Sweden) and $\beta$-caryophyllene (kindly supplied by Firmenich SA, Geneva, Switzerland). Equal amounts of these substances were mixed $(1 / 100)$ in hexane, to give a stock solution in all experiments. 1-Hexadecanol and geranylgeraniol (neat) were tested separately using the same methods as for the stock solution. As solvent, methanol or hexane (Merck, p.a. quality) was used. 3-Octanone (Sigma-Aldrich, Sweden) was used for the identification of volatiles of the fungi G. applanatum.

\section{Analytical equipment}

A HP 5890 gas chromatograph with a FID detector was used. Helium was used as the carrier gas, detector temp $250{ }^{\circ} \mathrm{C}$, injector temp $200{ }^{\circ} \mathrm{C}$, split/splitless injection for $30 \mathrm{~s}$. Column DB-5 (J\& W Scientific, Folsom, California, USA), $30 \mathrm{~m}$, id $0.25 \mathrm{~mm}$, film thickness $0.25 \mu \mathrm{m}$. Temperature program; $50{ }^{\circ} \mathrm{C}$ for $2 \mathrm{~min}, 10^{\circ} \mathrm{C} / \mathrm{min}$ to $280^{\circ} \mathrm{C}$, isothermal for $20 \mathrm{~min}$. Identifications were made with a Varian 3400 gas chromatograph connected to a Finnigan SSQ7000 mass spectrometer. Column DB-WAX (J\&W Scientific, Folsom, California, USA), $30 \mathrm{~m}$, id $0.25 \mathrm{~mm}$, film thickness $0.25 \mu \mathrm{m}$. Carrier gas helium at $69 \mathrm{kPa}$. Temperature program: $40{ }^{\circ} \mathrm{C}(1), 3{ }^{\circ} \mathrm{C} / \mathrm{min}$ up to $70^{\circ} \mathrm{C}, 15^{\circ} \mathrm{C} / \mathrm{min}$,

Table I. Physical properties of the compounds used.

\begin{tabular}{lccccc}
\hline Substance & $\begin{array}{c}\text { Compound } \\
\text { No. }\end{array}$ & $\begin{array}{c}\text { Retention time } \\
{[\mathrm{min}]}\end{array}$ & $\begin{array}{c}\text { Mol. } \\
\text { weight } \\
{[\mathrm{g} / \mathrm{mol}]}\end{array}$ & $\begin{array}{c}\mathrm{Bp} \\
{\left[{ }^{\circ} \mathrm{C}\right]}\end{array}$ & $\begin{array}{c}\mathrm{P}^{\mathrm{e}} \\
{[\mathrm{kPa}]}\end{array}$ \\
\hline a-Pinene & 1 & 7.48 & 136 & $155^{\mathrm{b}}$ & $6.6 \times 10^{-1}$ \\
1-Octen-3-ol & 2 & 8.22 & 128 & $173^{\mathrm{b}}$ & \\
Linalool & 3 & 10.42 & 154 & $194^{\mathrm{b}}$ & $5.8 \times 10^{-2}$ \\
cis-Verbenol & 4 & 11.25 & 152 & solid & \\
Terpinen-4-ol & 5 & 11.87 & 154 & $220^{\mathrm{b}}$ & \\
Verbenone & 6 & 12.42 & 150 & $227^{\mathrm{b}}$ & \\
Tridecane & 7 & 13.68 & 184 & $234^{\mathrm{b}}$ & $5.4 \times 10^{-3}$ \\
B-Caryophyllene & 8 & 15.77 & 204 & $262^{\mathrm{c}}$ & \\
Heptadecane & 9 & 19.11 & 240 & $302^{\mathrm{b}}$ & $3.6 \times 10^{-5}$ \\
E,E-Farnesol & 10 & 19.48 & 222 & $310^{\mathrm{b}}$ & \\
Ethyl hexadecanoate & 11 & 20.22 & 256 & $320^{\mathrm{b}}$ & $<1.0 \times 10^{-4}$ \\
1-Hexadecanol & 12 & 24.66 & 242 & $325^{\mathrm{b}}$ & $4.7 \times 10^{-7}$ \\
Geranylgeraniol & 13 & 290 & $420^{\mathrm{d}}$ & \\
\hline
\end{tabular}

a) Retention times found on the GC-column DB-5. b) Sigma-Aldrich (Stockholm, Sweden). c) Reference Conolly and Hill, 1991. d) Reference Costes, 1966. e) Vapour pressure (reference Dykyj and Repás, 1979). 
up to $200{ }^{\circ} \mathrm{C}(20)$. Injector temperature at $225^{\circ} \mathrm{C}$ and split/splitless injection was made for $30 \mathrm{~s}$. Total scan (TIC) m/z 30-300, $70 \mathrm{eV}$, scan rate $0.5 \mathrm{u} / \mathrm{s}$.

\section{Sorption materials}

A manual SPME holder (Supelco, Sweden) was used with two kinds of fibers. Coatings used were polydimethylsiloxane (PDMS) $100 \mu \mathrm{m}$ and carbowax/ divinylbenzene (CW/DVB) $65 \mu \mathrm{m}$.

Porapak Q 100/120 mesh, $200 \mathrm{mg}$ (Alltech, Scantech Lab AB, Partille, Sweden) and Tenax TA $80 / 100$ mesh, $100 \mathrm{mg}$ (Supelco, Sweden), were packed in glass vials (id $5 \mathrm{~mm}$ ) of the same kind. Charcoal, $20 \mathrm{mg}$ (CLSA-filter, Novart AG, Switzerland). The vials were vertically connected to the temperature controlled/regulated glass tube via an "L" tube (Fig. 1). The vial was in turn connected to the flow meter. After sampling, the ad- sorbents were extracted with hexane and $100 \mu \mathrm{l}$ portions were collected.

Methanol, $50 \mu$ l, was added to a U-tube before sampling (Fig. 1). The U-tube was dipped into a $79^{\circ} \mathrm{C}$ cooling bath $\left(\mathrm{CO}_{2} /\right.$ ethanol). At the end of the sampling the U-tube was rinsed with $50 \mu \mathrm{l}$ methanol. The total volume of methanol collected was $90 \mu \mathrm{l}$.

Air

The air was generated by an air generator (Chrompack International B. V., Middelburg, The Netherlands), and cleaned by passing through silica gel, active charcoal and a molecular sieve $(50 \mathrm{~nm})$. The gas flow was controlled by a flow meter (soap bubble meter) and the temperature in the glass tube was controlled using a thermostat and circulating water (Jursik et al., 1991).

A.

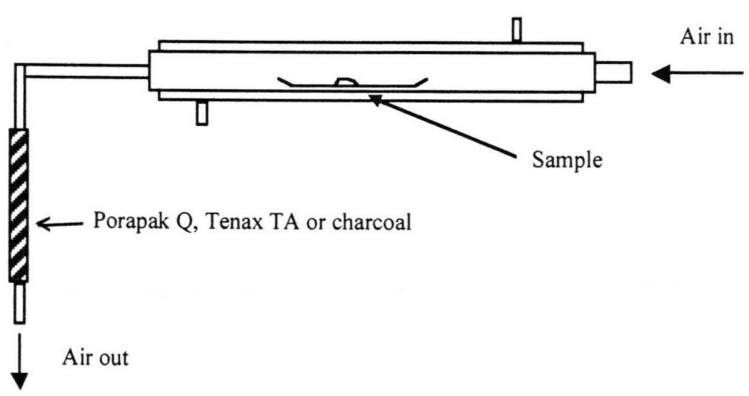

B.

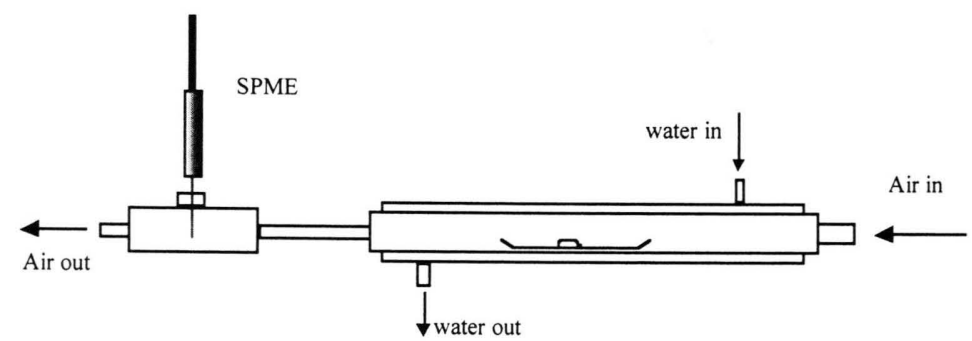

C.

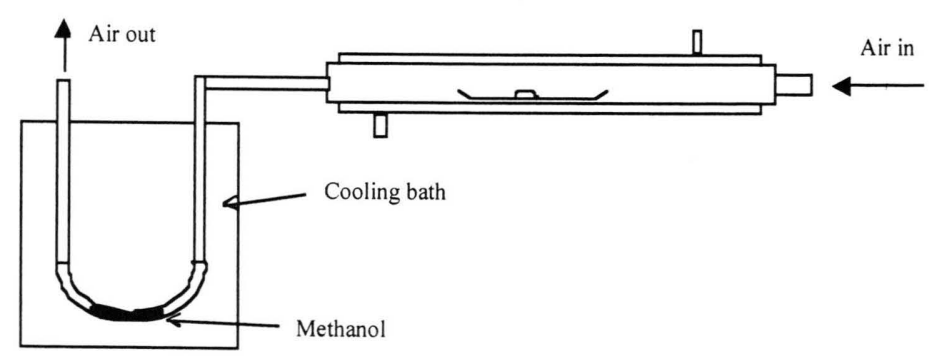

Fig. 1. System set-ups for the adsorption methods:

A. Purge \& Trap, B. SPME, C. Methanol. 


\section{Dynamic sampling of volatile compounds}

All samples were prepared through following procedure: The glassware (silanized) was washed with ethanol and dried by air. A previous sampling for $30 \mathrm{~min}$ with SPME (PDMS $100 \mu \mathrm{m}$ ) controlled the absence of compounds before extraction. A 10 $\mu \mathrm{l}$ capillary filled with the stock solution (approximately $100 \mu \mathrm{g}$ of each compound) was placed on a piece of aluminum foil $(5 \mathrm{~cm}$ long and $1 \mathrm{~cm}$ wide) and placed in the glass tube (inner volume $30 \mathrm{ml}$ ). The temperature was regulated to $27^{\circ} \mathrm{C}$ and the adsorbents were connected after the glass tube. The airflow generator was connected so as to inject clean air at a flow rate adjusted to $20 \mathrm{ml} /$ min, which was shown to be a suitable flow rate for all of the techniques used. The sampling time was 30 minutes for all methods and at least five replicates were made for each method $(n=5)$.

The various extraction set-ups, to be connected after the glass tube, are shown in Fig. 1. Before exposure, the SPME fibers were inserted into the glass tube through a screw cap with a Teflon septum and then exposed (Fig. 1B). Desorption was made for $2 \mathrm{~min}$, and followed by $10 \mathrm{~min}$ of cleaning at $200{ }^{\circ} \mathrm{C}$ before each new sampling. To verify that the amount of the compounds used was well within the operation range for all methods, test samples with $1 \mu \mathrm{l}$ of a $1 / 100$ of the stock solution (approximately $0.1 \mu \mathrm{g}$ of each compound) were brought into the glass tube. Collections were then made in duplicate, with each one of the adsorption methods in exactly the same way as described above.

\section{Storage of exposed SPME fibers}

The exposed fibers were kept in septum-sealed vials $(2 \mathrm{ml})$ at two temperatures $\left(6^{\circ} \mathrm{C}, 22^{\circ} \mathrm{C}\right)$. One fiber was left unprotected at the higher temperature $\left(22^{\circ} \mathrm{C}\right)$. Two periods of storage of exposed fibers were chosen, $1 \mathrm{~h}$ and 3 days. The samples were prepared by adding $10 \mu \mathrm{l}$ of $1 / 200$ of the stock solution in hexane to a $1 \mathrm{~cm}^{2}$ filter paper in a $7 \mathrm{ml}$ glass vial. The solvent was evaporated for $10 \mathrm{~s}$ and covered with a piece of aluminum foil. Sampling (static headspace) was done with a 100 $\mu \mathrm{m}$ PDMS SPME-fiber for $2 \mathrm{~min}$. Controls (starting values) were made by injecting/desorbing an exposed fiber into the GC directly after sampling.
The same fiber was then used for a new extraction before storage.

\section{Volatiles of a natural source}

The described and tested equipment above was also used on a natural source. Chopped fruiting bodies from the bracket fungi were chosen since it produces large amounts of 1-octen-3-ol, a key compound in environmental analyses. $8 \mathrm{~g}$ of $G$. applanatum fruiting body (chopped in $1 \mathrm{~cm}^{3}$ pieces) was placed in the sampling tube and the two SPME fibers used above was again tested here. Fruiting bodies of the fungus were collected $35 \mathrm{~km}$ north of Stockholm, Sweden and a deposit of a voucher specimen is stored on Royal Institute of Technology, Stockholm, Sweden. Tenax TA was used as purge and trap adsorbent. Sampling and collection was performed under the same conditions as for the standard mixture tested.

\section{Results and Discussion}

\section{Dynamic sampling}

Some of the properties of the organic compounds used are listed in Table I and their structures are shown in Fig. 2. Most of the extraction methods gave different results when used in a dynamic system (flow rate $20 \mathrm{ml} / \mathrm{min}$ ), Fig. 3, but the purge and trap methods (Porapak Q, Tenax TA and charcoal) and methanol (with a cold trap) gave the same results for the compounds extracted here. These extraction methods did not trap heptadecane, $E, E$-farnesol and ethyl hexadecanoate due to their low vapour pressure. They also gave more equal responses for the compounds than the SPME extractions. In general, solid phase microextraction gave a higher response for all compounds except for $\alpha$-pinene if calculated for 1 $\mu l$ volume, since the SPME fiber can be approximated with this volume (Pawliszyn, 1997). However when comparing the methods for the total amount extracted of the compounds, the purge and trap methods are by far more useful (Fig. 3b). From this (Fig. 3) one can see that SPME is more sensitive but when it comes to sample collection and preparation of samples for multipurpose use, the purge and trap methods is the best choice.

The two SPME fibers used (PDMS and CW/ DVB) also showed a big difference in between. 


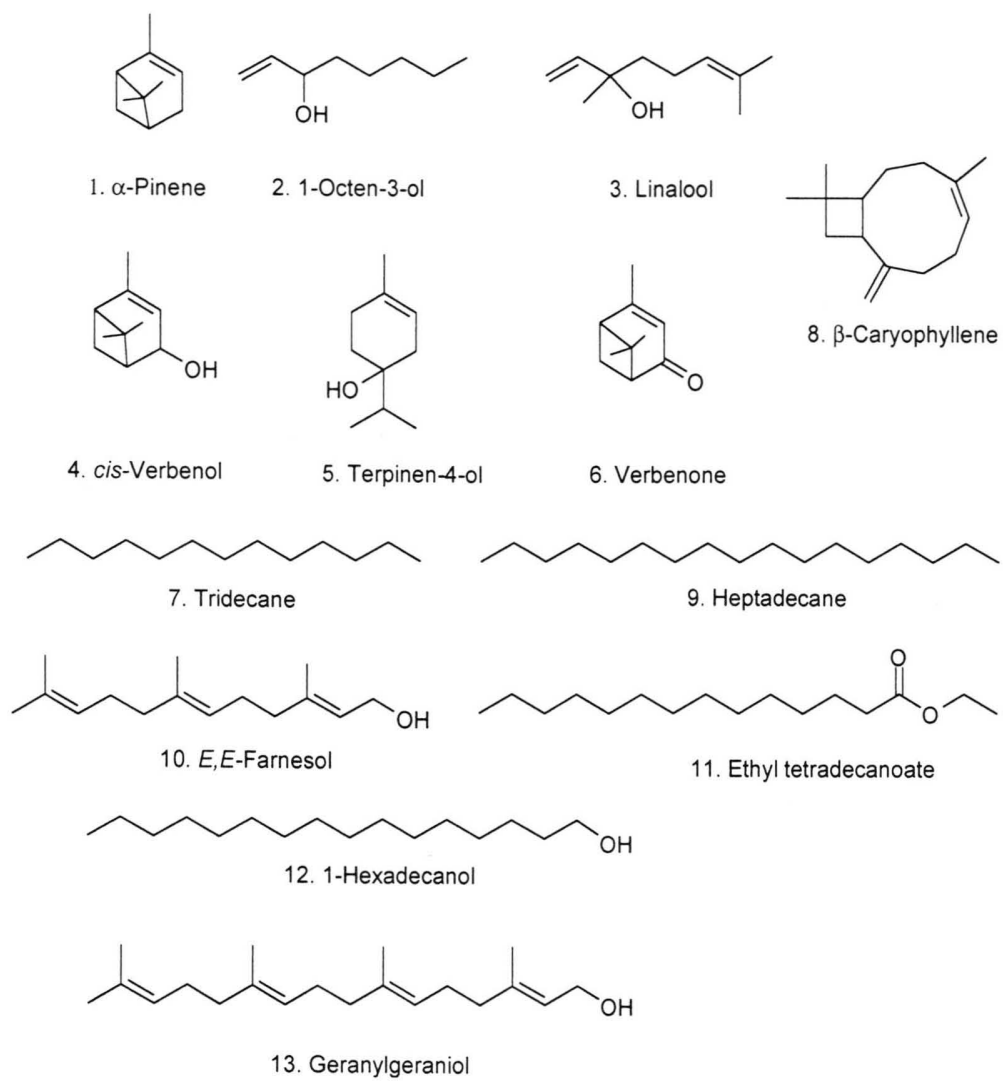

Fig. 2. Compounds used in the sampling experiments with the different adsorption methods.

The PDMS fiber was the only one to extract heptadecane, $E, E$-farnesol and ethyl hexadecanoate, while the CW/DVB fiber was better for extraction of the more polar and volatile compounds. The results from the sampling above the lower concentration $(1 \mu \mathrm{l}, 1 / 100$ of the stock solution) showed that all methods were compared within their range of detection for all compounds. The relative standard deviations for the methods (Table II) gave values ranging from 8 to $30 \%$, and within the range for comparing the methods.

1-Hexadecanol and geranylgeraniol were not extracted by any of the methods and are, therefore, not shown in any of the figures or in the results. However, the results are still interesting since they (may) indicate the limit of substances detectable by headspace at room temperature, airflow used and with analytical equipment employed.

It is clear that, the purge and trap and SPME methods extract compounds in different amounts.
It is useful to know the range of volatility of the expected compounds when selecting the best technique. Considering the easy handling of SPME, its efficiency and sensitivity, it is the best choice for all of the compounds analyzed here except $\alpha$-pinene, when performing a qualitative analysis. However, it is more difficult to make proper quantification with this method than with liquid extractions. Another disadvantage is that an SPME sample can be used only once. In the purge and trap methods several analyses can be made from the same extract. The dynamic sampling with SPME may also favour the heavier compounds, which have a low vapour pressure, compared to static sampling with the same type of fiber. The more volatile compounds, e.g. $\alpha$-pinene, were not favoured by the air stream with SPME and were probably desorbed from the fiber coating as easily as they were adsorbed. The net result was that $\alpha$-pinene, was not extracted at all. When using an air stream with SPME this effect must be considered in work 


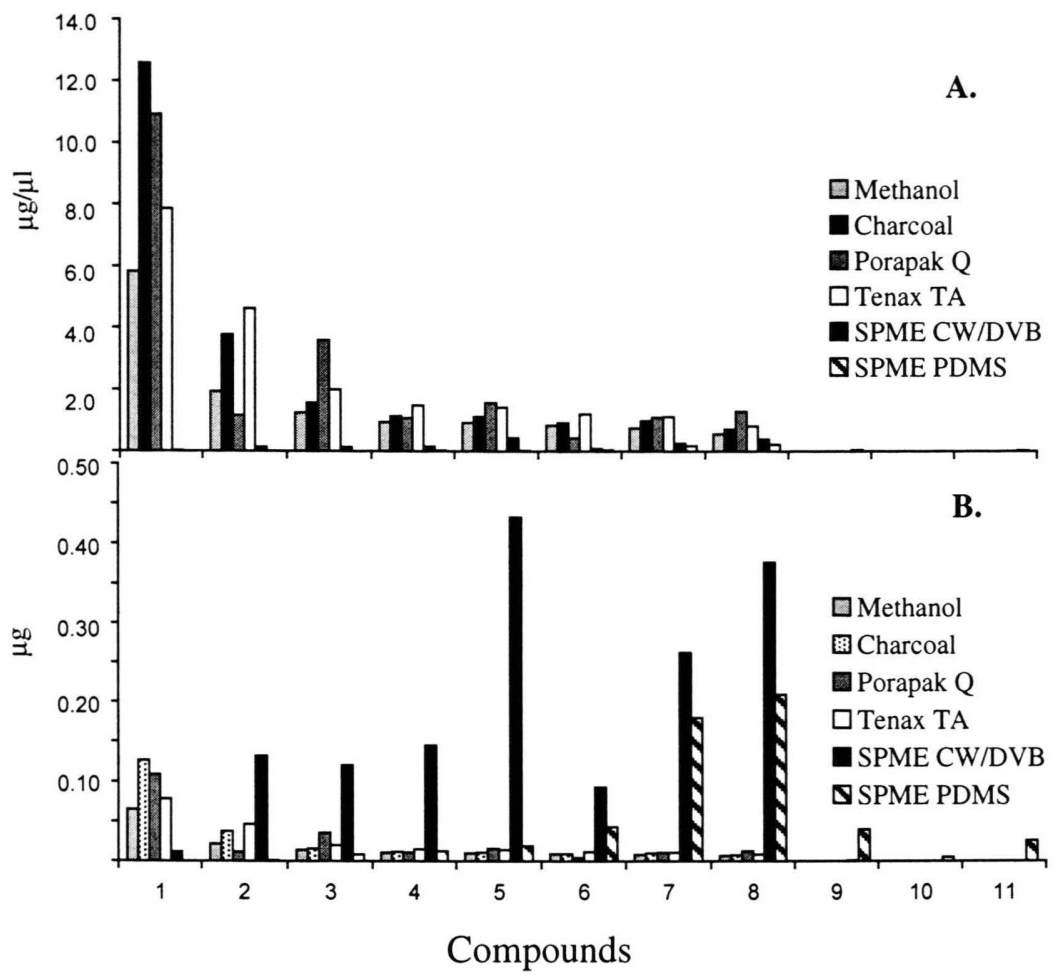

Fig. 3. Comparison of headspace techniques in an air stream. The $\mathrm{x}$-axis corresponds the compounds according to Fig. 2. A) Extracted amount, based on $1 \mu$ l injections. B) Total amount extracted.

Table II. Relative Standard Deviations obtained with the various desorption techniques (5 replica).

\begin{tabular}{|c|c|c|c|c|c|c|}
\hline $\begin{array}{l}\operatorname{RSD}[\%] \\
(n=5)\end{array}$ & Charcoal & Methanol & Porapak $Q^{a}$ & Tenax $\mathrm{TA}^{\mathrm{b}}$ & $\begin{array}{c}\text { SPME } \\
\mathrm{CW}^{-\mathrm{DVB}}{ }^{\mathrm{c}}\end{array}$ & $\begin{array}{l}\text { SPME } \\
\text { PDMS }^{d}\end{array}$ \\
\hline$\alpha$-Pinene & 18 & 22 & 20 & 16 & 15 & - \\
\hline 1-Octen-3-ol & 15 & 29 & 21 & 15 & 20 & 16 \\
\hline Linalool & 21 & 17 & 12 & 12 & 7 & 9 \\
\hline cis-Verbenol & 25 & 11 & 14 & 13 & 23 & 38 \\
\hline Terpinen-4-ol & 25 & 13 & 17 & 10 & 6 & 24 \\
\hline Verbenone & 27 & 12 & 21 & 11 & 11 & 16 \\
\hline Tridecane & 25 & 9 & 19 & 18 & 6 & 12 \\
\hline$\beta$-Caryophyllene & 26 & 5 & 21 & 24 & 7 & 8 \\
\hline Heptadecane & - & - & - & - & 19 & 28 \\
\hline$E, E$-Farnesol & - & - & - & - & - & 17 \\
\hline Ethyl hexadecanoate & - & - & - & - & - & 26 \\
\hline
\end{tabular}

a Porapak Q, a porous polymer based on divinylbenzene, 100/120 mesh, $200 \mathrm{mg}$ (Alltech, Scantech Lab AB, Partille, Sweden). ${ }^{b}$ Tenax TA, a porous polymer based on 2,6-diphenyl-p-phenylene oxide, 80/100 mesh, $100 \mathrm{mg}$ (Supelco, Sweden). ${ }^{c}$ Solid-Phase Microextraction (SPME), coated with carbowax/divinylbenzene (CW/DVB) $65 \mu \mathrm{m}$ (Supelco, Sweden). ${ }^{d}$ Solid-Phase Microextraction (SPME), coated with polydimethylsiloxane (PDMS) $100 \mu \mathrm{m}$ (Supelco, Sweden).

with volatile substances. Dynamic sampling may be of importance when screening for larger organic molecules, such as heptadecane or ethyl hexadecanoate. For the extraction of volatile com- pounds at trace levels with SPME, the Carbowax/ divinylbenzene $65 \mu \mathrm{m}$ fiber was outstanding among the methods used. 


\section{Storage of exposed SPME fibers}

The storage of exposed fibers in different environments for different periods of time showed that SPME fibers could be stored for several days under proper conditions. The results obtained after storage depended on the method, the temperature, the time and the character of the substance itself. As expected and as shown in Fig. 4, volatile compounds were the most difficult ones to store in a fiber, whereas the heavier semi-volatile compounds were more easily kept in the fiber for a longer time. The best conditions for storing the fibers were low temperatures and in sealed vials. When the fibers were left unprotected in the laboratory at $22{ }^{\circ} \mathrm{C}$ the loss of the most volatile sub- stances was remarkable as early as after one hour. Another effect of storing fibers in the open (fiber tip still retracted in the tube) was that other substances were adsorbed from the surrounding air. That way of leaving fibers in the open has actually been used for air monitoring, and is called in-tube sampling (Pawliszyn, 1997). The risk of contamination observed, showed the importance of proper cleaning and storage of fibers before use, especially if they are to be used for sampling elsewhere or later in time.

\section{Collection of volatiles from $G$. applanatum}

The CW/DVB fiber extracted the highest amount of 1-octen-3-ol and 3-octanone, when

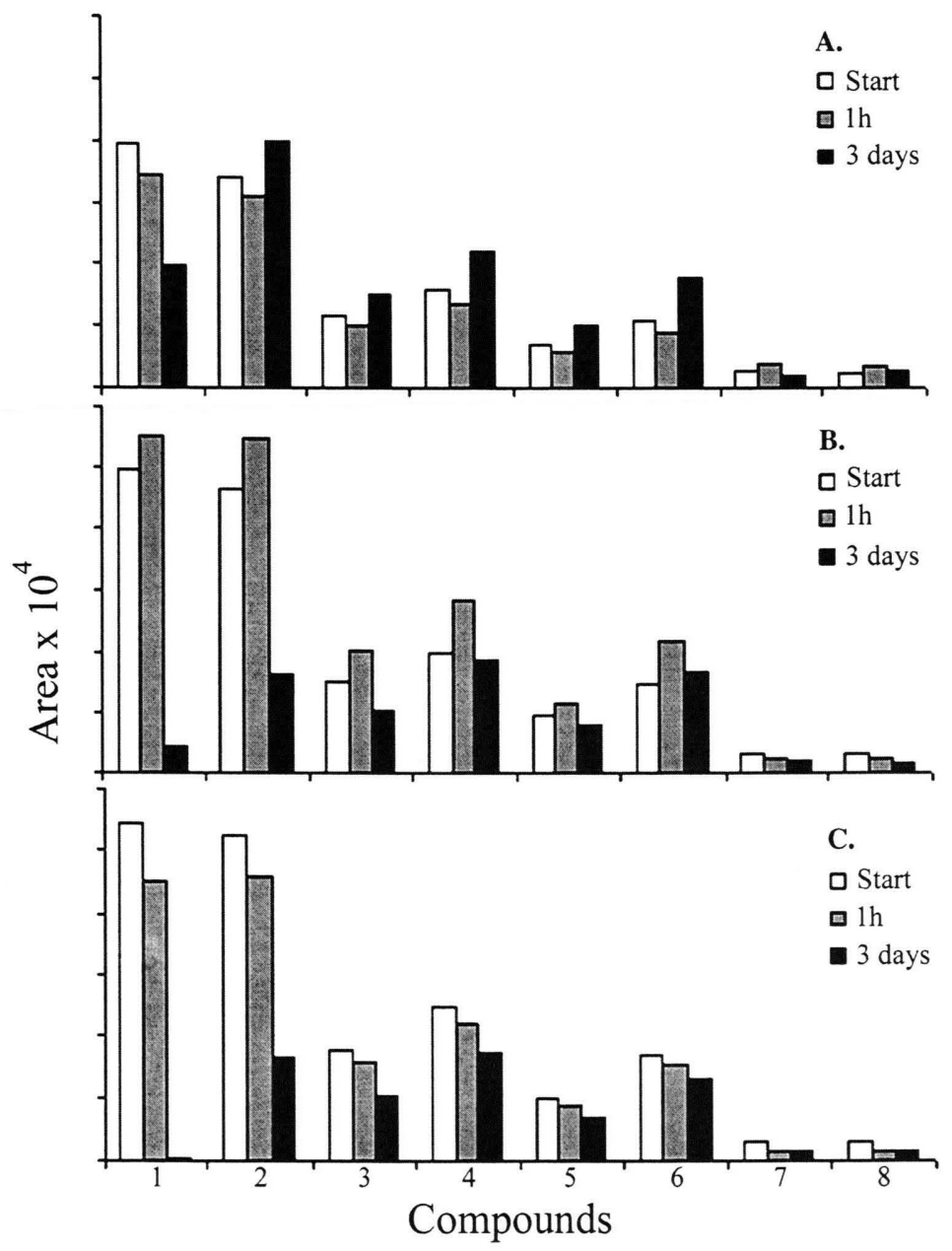

Fig. 4. Storage of exposed fibers. The x-axis corresponds the compounds according to Fig. 2 and the y-axis corresponds the GC response area. A) Sealed in $6{ }^{\circ} \mathrm{C}, \mathrm{B}$ ) Sealed in $\left.22^{\circ} \mathrm{C}, \mathrm{C}\right)$ Unprotected in $22^{\circ} \mathrm{C}$. 


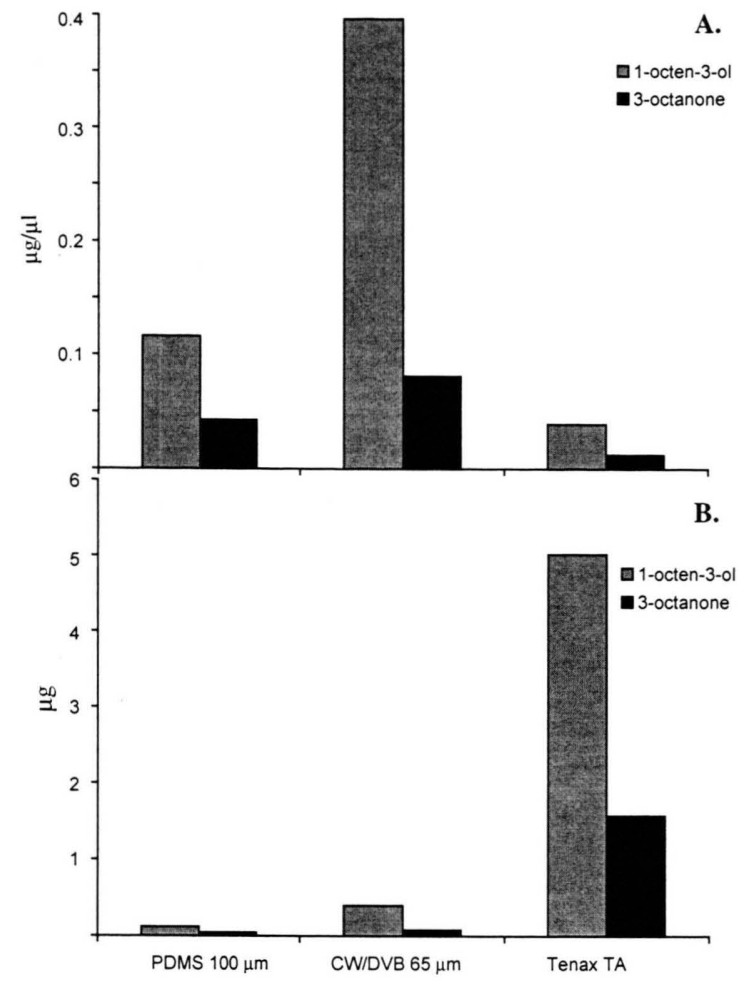

Fig. 5. Sampling of fresh G. applanatum (8 g chopped in $1 \mathrm{~cm}^{3}$ pieces) for 30 minutes using the dynamic setup with the different sorption/collection methods (SPME PDMS, SPME CW/DVB and Tenax T. A) Amount extracted from $8 \mathrm{~g} \mathrm{G}$. applanatum based on $1 \mu \mathrm{l}$ injections. B) Total amount extracted from $8 \mathrm{~g} \mathrm{G}$. applanatum.

using the dynamic sampling set-up with SPME (PDMS and CW/DVB fibers) and Tenax TA for collection of volatiles from a natural source (in this case G. applanatum (Fig. 5a and b). These metabolites were identified by comparison of their retention times, with authentic standards. However this result is only valid when comparing the 1 $\mu \mathrm{l}$ injections, which has also been discussed above. When calculating the total amount extracted (Fig. 5b), Tenax is by far the best sampling method. SPME is however still the most sensitive method for a qualitative analysis (as shown when using the standard mixture above).

Both of the compounds found have been shown to be important in the attraction of insects to fungi (Fäldt et al., 1999). Headspace samples from natural sources (e.g. insects, plants, fungi etc) have been shown to emit complex mixtures. In studies including insect-plant relationships we stress the importance of choosing the appropriate combination of sampling methods, and if possible, optimize the sampling by using both headspace SPME and purge and trap techniques.

\section{Acknowledgements}

We thank Dr. Karel Stránsky for providing the special glass equipment used for the airflow samplings and Dr. Gunhild Aulin-Erdtman for valuable discussions. This study was financed by the Royal Swedish Academy of Engineering Sciences (IVA), the Czech Academy of Sciences, the Grant Agency of the Czech Republic (grant number: 203/98/0453), the Swedish Council for Forestry and Agricultural Research (SJFR) and the Center for Environmental Science, KTH, the Royal Institute of Technology. 
Agelopoulos N. G. and Pickett J. A. (1998), Headspace analysis in chemical ecology: effects of different sampling methods on ratios of volatile compounds present in headspace samples. J. Chem Ecol. 24, 1161-1172.

Bartelt R. J. and Zilkowski B. W. (1999), Nonequilibrium quantitation of volatiles in air streams by solid phase microextraction. Anal. Chem. 71, 92-101.

Borg-Karlson A.-K. (1990), Chemical and ethological studies of pollination in the genus Ophrys (Orchidaceae). Phytochemistry 29, 1359-1387.

Borg-Karlson A.-K. and Mozuraitis R. (1996), Solid phase microextraction technique used for collecting semiochemicals. Identification of volatiles released by individual signalling Phyllonorycter sylvella moths. Z. Naturforsch. 51c, 599-602.

Conolly J. D. and Hill R. A. (1991), Dictionary of terpenoids. Vol 1. Mono- and Sesquiterpenoids. $1^{\text {st }}$ ed Chapman and Hall, London.

Costes C. (1966), Biosynthèse du phytol des chlorophylles et du squelette tétraterpénique des carotenoïdes dans les feuilles vertes. Phytochemistry 5, 311-324.

Cross J. H., Byler R. C., Cassidy R. F., Silverstein R. M., Greenblatt R. E., Burkholder W. E., Levinson A. R., and Levinson H. Z. (1976), Porapak-Q collection of pheromone components and isolation of $(Z)$ - and $(E)$ methyl-8-hexadecenal, sex pheromone components, from the females of four species of Trogoderma (Coleoptera: Dermestidae). J. Chem. Ecol. 2, 457-468.

Dykyj J. and Repás M. (1979), Tlak nasytenej pary organickych zlúcenín. Vol. I and II. Veda, Bratislava.

Eisert R., Pawliszyn J., Barinshteyn G., and Chambers D. (1998), Design of an automated analysis sytem for the determination of organic compounds in continuous air stream using solid-phase microextraction. Anal. Comm. 35, 187-189.
Eriksson M., Swartling A., Fäldt J., Borg-Karlson A.-K., and Dalhammar G. (1998), Biological degradation of diesel fuel in water and soil monitored with solid phase microextraction and GC-MS. Appl. Microbiol. Biotechnol. 50, 129-134.

Fäldt J., Jonsell M., Nordlander G., and Borg-Karlson A.-K. (1999), Volatiles of bracket fungi Fomitopsis pinicola and Fomes fomentarius and their functions as insects attractants J. Chem. Ecol. 25, 567-590.

Jursik T., Stránsky K., and Ubik K. (1991), Trapping system for trace organic volatiles. J. Chromatogr. 586, 315-322.

Knudsen J. T., Tollsten L., and Bergström L. G. (1993), Floral scents-a checklist of volatile compounds isolated by headspace techniques. Phytochemistry 33, 253-280

Malosse C., Ramirez-Lucas P., Rochat D., and Morin J.P. (1995), Solid phase microextraction, an alternative method for the study of airborne insect-pheromones (Metamasius hemipterus, Coleoptera, Curculionidae). J. High. Resol. Chromatogr. 18, 669-670.

Matich A. J., Rowan D. D., and Banks N. H. (1996), Solid phase microextraction for quantitative headspace sampling of apple volatiles. Anal. Chem. 68, 4114-4118.

Miller M. E. and Stuart J. D. (1999), Comparison of gassampled and SPME sampled static headspace for the determination of volatile flavor components. Anal. Chem. 71, 23-27.

Pawliszyn J. (1997), Solid Phase Microextraction Theory and Practise, Wiley-WCH: New York. 